\title{
Idiopathic Sclerosing Mesenteritis: An Extremely Rare Cause of Mesenteric Mass
}

\author{
Samyak Dhruva, d, Meena Kashi ${ }^{\mathrm{b}}$, Dhwani Pandya ${ }^{\mathrm{c}}$
}

\begin{abstract}
Idiopathic sclerosing mesenteritis (ISM) is an extremely rare condition in which mesenteric adipose tissue undergoes necrotic and fibrotic changes. It is also known as "retractile mesenteritis", "mesenteric panniculitis" or "fat necrosis of the mesentery". Most cases are seen in Caucasian males between the fifth and seventh decades of life. Some of the suggested causes of this extremely rare condition include autoimmune disorders, abdominal trauma, malignancy, abdominal infections and even IgG4-related diseases; however, the actual cause remains unknown. Most cases are either self-limiting or improve with medical therapy. However, aggressive cases requiring emergent surgical interventions to relieve intestinal obstruction have been reported in the literature. We present a case of a 60 -year-old male patient who presented to the emergency room with severe abdominal pain and was diagnosed with ISM that required surgical intervention to relieve the symptoms.
\end{abstract}

Keywords: Idiopathic sclerosing mesenteritis; Fat necrosis; Mesenteric mass; Retractile mesenteritis; Panniculitis

\section{Introduction}

Sclerosing mesenteritis is an extremely rare inflammatory process that predominantly affects the mesenteric adipose tissue. It was first described in 1924 by Jura as "retractile mesenteritis" [1]. Several terms have been used since then to describe different levels of adipose tissue involvement like mesenteric lipodystrophy (adipocyte necrosis), mesenteric panniculitis (chronic inflammatory state of the adipose tissue) and sclerosing mesenteritis (fibrosis of adipose tissue) [2]. Histological continuation

Manuscript submitted October 31, 2021, accepted November 26, 2021

Published online December 2, 2021

aDepatment of Internal Medicine, Medstar St. Mary's Hospital, Leonardtown, MD, USA

bDepatment of Pathology, Staten Island University Hospital, Staten Island, NY, USA

'Depatment of Internal Medicine, Staten Island University Hospital, Staten Island, NY, USA

${ }^{\mathrm{d} C o r r e s p o n d i n g ~ A u t h o r: ~ S a m y a k ~ D h r u v, ~ D e p a t m e n t ~ o f ~ I n t e r n a l ~ M e d i c i n e, ~}$ Medstar St. Mary's Hospital, Leonardtown, MD, USA.

Email: samyakdhruv92@gmail.com

doi: https://doi.org/10.14740/jmc3825 is not documented but generally, patients have one predominant feature depending on adipose tissue involvement. By 2016, only 300 cases had been reported in the literature, showing the rarity of the clinical entity [3]. It is mostly seen in Caucasian males between the fifth and seventh decades of life [2]. Several causal factors are suggested like autoimmune disorders, abdominal trauma, malignancy, abdominal infections and even IgG4-related diseases, but the actual cause remains unknown [4].

\section{Case Report}

\section{Investigations}

A 60-year-old man with the past medical history of hypertension and diabetes presented to the hospital with diffuse cramping abdominal pain that started 3 weeks before his presentation and worsened on the day of admission. The pain was non-radiating without any worsening or relieving factors.

The pain was not related to food or bowel movements. He did endorse $30 \mathrm{lbs}$ weight loss over the last 3 months as well as loss of appetite. He denied any night sweats, fever, chills, dysuria, diarrhea, constipation, nausea or vomiting. In the emergency room (ER), temperature was $36.8^{\circ} \mathrm{C}$, heart rate was $86 /$ min, blood pressure was $164 / 78 \mathrm{~mm} \mathrm{Hg}$, respiratory rate was 18 / min, and oxygen saturation was $100 \%$. Laboratory studies were unremarkable except white blood cell count of 14,000 cells $/ \mu \mathrm{L}$.

\section{Diagnosis}

Computed tomography (CT) scan of the abdomen showed a $7.2 \times 6.4 \times 3.3 \mathrm{~cm}$ hypodense amorphous collection versus mass within mesentery, with the possibility of the necrotic tumor (Fig. 1). General surgery, gastroenterology and interventional radiology teams were consulted for a multidisciplinary approach. Owing to the proximity of the transverse colon and stomach to the mass, it was decided to proceed with endoscopy (EGD) with endoscopic ultrasound (EUS)-guided biopsy and colonoscopy. EGD was suggestive of an irregular hypoechoic mass measuring $3.7 \mathrm{~cm}$ in its longest axis outside the gastric echo layer from a distal body position (Fig. 2). Ultrasoundguided fine-needle biopsy (FNB) was performed with four passes from the distal body of the stomach. The EUS view of the pancreas, left kidney, adrenal, spleen, liver lobes and portal vein was unremarkable. The common bile duct and pancreatic 


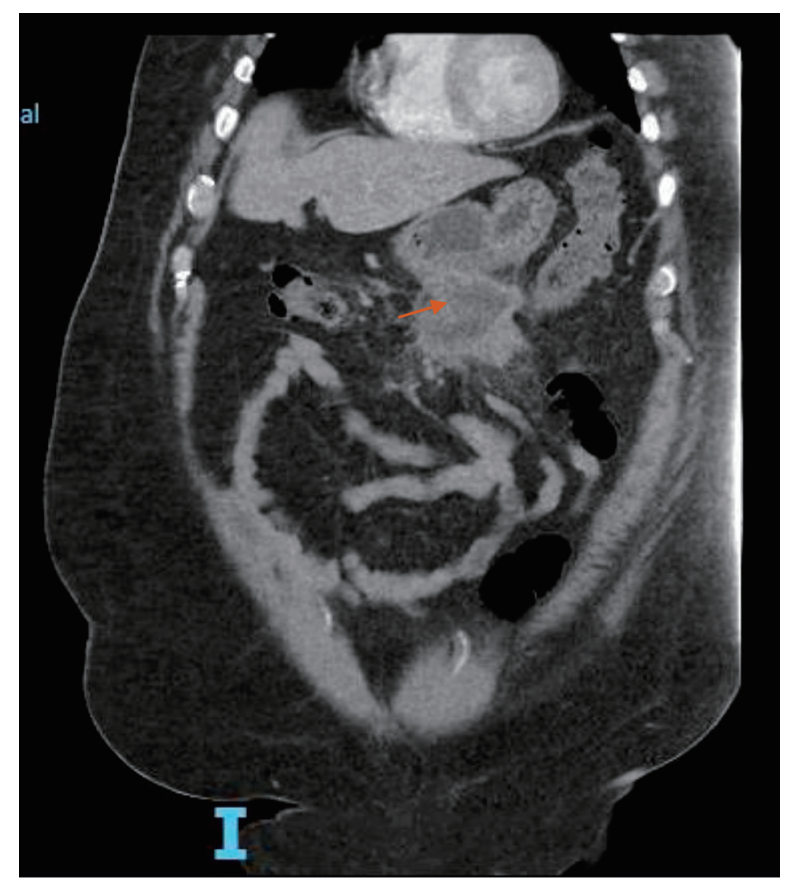

Figure 1. A coronal section of abdomen computed tomography with intravenous contrast demonstrating a rim enhancing hypodense amorphous lesion in mesentery. This lesion is contiguous with the inferior aspect of the greater curvature of the stomach as well as a loop of bowel. Also surrounding the lesion, an extensive fat stranding was noted (orange arrow).

duct were not dilated. Colonoscopy showed external and internal hemorrhoids. At descending colon, there was an irregular $8 \mathrm{~mm}$, polypoid, granular appearing lesion with surrounding edema likely due to diverticulitis or the mesenteric mass eroding the colon. Cold forceps biopsies were taken from that area. Also, the area around it was marked by India Ink preparation for the surgical references during laparotomy if needed. The terminal ileum showed no abnormalities. Biopsy results of the EUS and colonoscopy were non-specific.

\section{Treatment}

Due to the non-specific nature of biopsies and continued pain, diagnostic and therapeutic laparoscopy was performed which showed a large abscess cavity in the base of transverse colon, mesentery, and the loop of small bowel. Abscess cavity was drained with removal of inflammatory mass and necrotic tissue, and small bowel was separated. Culture results were negative for any infection. Histopathology analysis of the surgical specimen confirmed the mass as extensive fat necrosis and nodules in the mesentery suggestive of sclerosing mesenteritis (Fig. 3).

\section{Follow-up and outcomes}

His abdominal pain resolved postoperatively, and he was discharged with the outpatient follow-up with our surgical team and primary care provider.

\section{Discussion}

Sclerosing mesenteritis is an inflammatory disorder of the in-

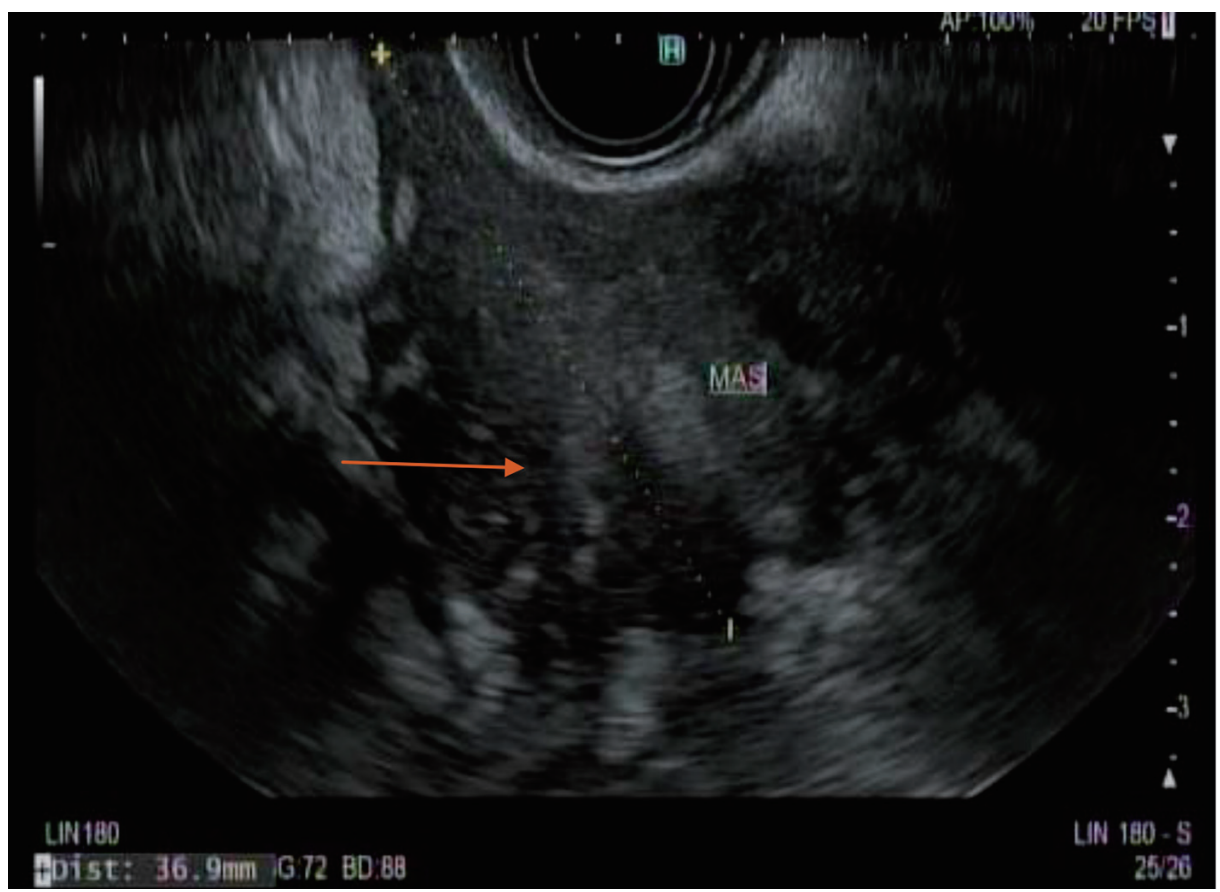

Figure 2. A Linear echo endoscopic image showing irregular hypoechoic mass of size $3.7 \mathrm{~cm}$ (orange arrow) outside the gastric echo layer in distal gastric body. 

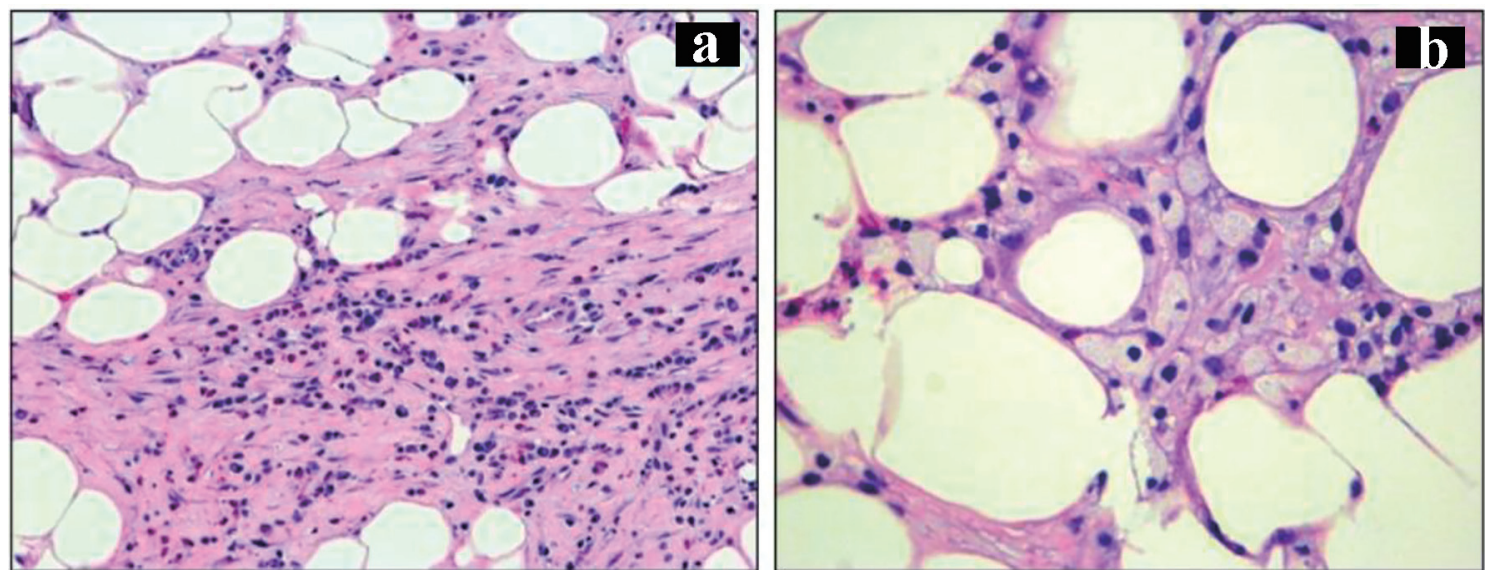

Figure 3. (a) Fat necrosis, area of fibrosis and infiltration of chronic inflammatory cells (H\&E, magnification, $\times 20)$. (b) Infiltration of lipid laden macrophages (H\&E, magnification, $\times 40)$. H\&E: hematoxylin and eosin stain.

testinal mesentery. As mentioned above, it is more common in white men in fifth to seventh decade of life. Most cases are asymptomatic. When symptomatic, abdominal pain is the most common symptom [5]. Occasionally patients have nausea and vomiting which may suggest intestinal obstruction [6]. Blood work helps in ruling out other differentials of abdominal pain and may suggest underlying etiology of the sclerosing mesenteritis. CT scan of the abdomen is the best imaging modality to investigate this condition where it is generally described as a heterogeneous solitary mass, mainly localized in the mesentery, usually left-sided, with delocalization of the surrounding structures. The two main radiological hallmark signs are fat ring sign, described as a ring of normal fat around vessels and lymph nodes and pseudo-capsule sign, defined as a thin fibrotic rim around the mass. They are identified in $70-92 \%$ and $50-60 \%$ of the patients, respectively [7]. Definitive diagnosis is made by histopathological analysis of the biopsy specimen. It may show fibrosis, inflammation, and fat necrosis and occasionally lipid-laden foamy macrophages may be found infiltrating the mesenteric fat [4]. Histopathology also helps in ruling out peritoneal carcinomatosis which might have similar CT appearance as sclerosing mesenteritis [8]. Before terming it as "idiopathic sclerosing mesenteritis", common causes like autoimmune diseases, abdominal trauma, and IgG4-related diseases need to be excluded [9]. Literature review suggests these patients respond to the combination of corticosteroid and tamoxifen when symptomatic in most cases [10]. Other immunosuppressive agents such as infliximab, cyclosporine and azathioprine have also been used with successful outcomes [11]. Most cases slowly progress and may resolve over time. Though, extremely aggressive cases requiring emergency surgeries to relieve the intestinal obstruction are also published in the literature [6]. Overall, most patients will improve over time and if it is secondary to autoimmune conditions, the treatment of the autoimmune condition improves the inflammation in the mesentery. More detailed studies are needed to identify the definite etiology of sclerosing mesenteritis. Randomized clinical trials on the appropriate treatment strategies and how long the immunosuppression must be continued are lacking due to the rarity of the disease.

\section{Conclusions}

Sclerosing mesenteritis is a rare but important differential of a patient with abdominal pain and mesenteric mass on imaging. Idiopathic sclerosing mesenteritis is a diagnosis of exclusion after ruling out some important causes like autoimmune diseases and malignancy and is usually confirmed by tissue diagnosis. Tamoxifen and prednisone have been used for treatment with successful outcomes in most cases. In some rare cases, diagnosis remains in doubt even after endoscopic biopsies like our case, in such cases laparoscopic resection of the mesenteric mass provides tissue for the diagnosis and also relieves the pain the discomfort of the patient. Further research is needed to establish the etiology of the disease and proper treatment protocols including medical vs. surgical management and their respective outcomes.

\section{Acknowledgments}

None to declare.

\section{Financial Disclosure}

None to declare.

\section{Conflict of Interest}

None to declare.

\section{Informed Consent}

Informed patient consent was obtained to write and publish this case report. 


\section{Author Contributions}

Samyak Dhruv wrote and edited the manuscript. Meena Kashi and Dhwani Pandya helped in editing.

\section{Data Availability}

The authors declare that data supporting the findings of the study are available within the article.

\section{References}

1. Hussein MR, Abdelwahed SR. Mesenteric panniculitis: an update. Expert Rev Gastroenterol Hepatol. 2015;9(1):6778.

2. Rey A, Busnelli VMC, Santino JP, Van Domselaar F, Quintana GO, Seehaus AC, De Paula JA. Extensive mesenteric lipodystrophy of the left colon: case report and brief review of the literature. Acta Gastroenterol Latinoam. 2016;46(1):43-47.

3. Kgomo M, Elnagar A, Mashoshoe K. Mesenteric panniculitis. BMJ Case Rep. 2017;2017; bcr-2017-220910.

4. Burns RL, Bhavnagri SJ. Undiagnosed Sjogren's syndrome presenting as mesenteric panniculitis. Case Rep
Rheumatol. 2016;2016:7207638.

5. Green MS, Chhabra R, Goyal H. Sclerosing mesenteritis: a comprehensive clinical review. Ann Transl Med. 2018;6(17):336.

6. Madubogwu CI, Okani CO. Sclerosing mesenteritis: A case of acute abdomen and intestinal obstruction. Niger J Med. 2016;25(1):86-89.

7. Buragina G, Magenta Biasina A, Carrafiello G. Clinical and radiological features of mesenteric panniculitis: a critical overview. Acta Biomed. 2019;90(4):411-422.

8. Watanabe T, Terai S, Tsukada T, Takeshita M, Matsui K, Amaya K, Kaji M, et al. Sclerosing mesenteritis mimicking metachronous peritoneal metastases from descending colon adenocarcinoma. World J Surg Oncol. 2017;15(1):142.

9. Kelly JK, Hwang WS. Idiopathic retractile (sclerosing) mesenteritis and its differential diagnosis. Am J Surg Pathol. 1989;13(6):513-521.

10. Akram S, Pardi DS, Schaffner JA, Smyrk TC. Sclerosing mesenteritis: clinical features, treatment, and outcome in ninety-two patients. Clin Gastroenterol Hepatol. 2007;5(5):589-596; quiz 523-584.

11. Rothlein LR, Shaheen AW, Vavalle JP, Smith SV, Renner JB, Shaheen NJ, Tarrant TK. Sclerosing mesenteritis successfully treated with a TNF antagonist. BMJ Case Rep. 2010;2010:bcr.07.2010.3145. 